\title{
¿Existe asociación entre la morfología normal del espermatozoide y su cinética de desplazamiento?
}

\author{
Munuce $\mathrm{MJ}^{*, * *}$, Cardona-Maya $\mathrm{W}^{* * *}$, Berta $\mathrm{CL}^{*, * *}$. \\ *Reprolab - Sanatorio Británico de Rosario, Argentina. ${ }^{* *}$ Servicio de Reproducción Humana y Planificación \\ Familiar, Cátedra de Ginecología, Universidad Nacional de Rosario, Argentina. ${ }^{* * *}$ Becario del Programa \\ Latinoamericano de Capacitación e Investigación en Reproducción Humana - PLACIRH.
}

Actas Urol Esp 2006; 30 (6): 591-597

\begin{abstract}
RESUMEN
¿EXISTE ASOCIACIÓN ENTRE LA MORFOLOGÍA NORMAL DEL ESPERMATOZOIDE Y SU CINÉTICA DE DESPLAZAMIENTO?

Objetivo: Evaluar si existe asociación entre el \% de formas normales y la cinética de desplazamiento en espermatozoides humanos.

Material y métodos: Se analizaron en forma retrospectiva los espermogramas de 203 pacientes evaluados mediante la ayuda de un sistema computarizado de análisis de movimiento. La evaluación de la morfología se realizó en forma micrométrica según el criterio estricto. Sólo se incluyeron los pacientes que presentaron $>20$ x $106 \mathrm{esp} / \mathrm{ml}$ y $>50 \%$ de motilidad progresiva. Los datos de 168 pacientes fueron divididos según el \% de formas normales iniciales en tres grupos a) $<4 \%(n=22)$, b) entre $4-13 \%(89 \%)$ y c) $\geq 14 \%(n=57)$ y se compararon los datos obtenidos. Con el objeto de seleccionar espermatozoides móviles, 35 muestras fueron tratadas con un gradiente discontinuo, evaluándose tanto el \% de formas normales como los parámetros de movilidad basales y luego de la selección.

Resultados: El análisis cinético mostró que tanto la concentración, el \% de espermatozoides móviles y rápidos (>25 $\mu \mathrm{m} / \mathrm{s}$ ), así como la velocidad media (VAP) y el desplazamiento de la cabeza (LHD) se vieron incrementados con la cantidad de espermatozoides morfológicamente normales presentando los valores más bajos en el grupo $<4 \%$ respecto al grupo $\geq 14 \%$, ( $p<0,05$ ). La LIN se mantuvo constante entre los grupos. En las muestras seleccionadas se incrementaron significativamente las variables cinéticas así como la morfología $(\mathrm{p}<0,0001)$. Los resultados muestran que aún en el caso de muestras teratozooespérmicas la utilización de gradientes mejora significativamente el \% de formas normales respecto de los valores basales $(\mathrm{p}<0,0002)$.

Conclusiones: Nuestros resultados apoyarían la hipótesis de que los espermatozoides morfológicamente mejores serían aquellos que presentan mejores características de movimiento medido en forma objetiva. De este modo podriamos suponer que in vivo, los mejores espermatozoides formarian parte de una "élite" en la carrera hacia el sitio de fecundación.
\end{abstract}

Palabras clave: Espermatozoide. Morfología. Criterios estrictos. Cinética de desplazamiento. Gradiente de densidad.

\section{ABSTRACT}

IS THERE AN ASSOCIATION BETWEEN SPERM NORMAL MORPHOLOGY AND THEIR KINETIC DISPLACEMENT?

Objective: To evaluate if there exist an association between the \% of normal forms and the kinetic characteristics in human spermatozoa

Material and Methods: A retrospective study was performed to analyze semen samples of 203 patients by Computer Assisted Semen Analysis. Sperm morphology was evaluated by the aid of a micrometric objective according to strict criteria. Only the patients presenting $>20 \times 106 \mathrm{sperm} / \mathrm{ml}$ and $>50 \%$ of progressive motility were included. Data from 168 patients were divided according to the $\%$ of normal forms in three groups a) $<4 \%(n=22)$, b) between $4-13 \%(n=89)$ and c) $\geq 14 \%$ ( $n=57$ ). Data collected among groups were compared. In order to select a motile sperm population 35 samples were treated by a discontinuous gradient and the $\%$ of normal forms as well as motility parameters evaluated before and after selection.

Results: The kinetic analysis showed that sperm concentration, the \% of motile and rapid spermatozoa $(>25 \mu \mathrm{m} / \mathrm{s})$ as well as the average path velocity (VAP) and the lateral head displacement (LHD) were increased in association with the $\%$ of normal spermatozoa presenting the lowest values in the group $<4 \%$ with respect to the $\geq 14 \%$ group, (p<0.05). Linearity (LIN) remained constant among groups. Kinetic parameters and sperm morphology were significantly increased $(\mathrm{p}<0.0001)$ in the selected samples Results showed that the use of gradients even in teratozoospermic samples improves significantly the \% of normal forms respect to baseline values $(\mathrm{p}<0.0002)$.

Conclusions: Our results would support the hypothesis that morphologically better spermatozoa would be associated with those with better movement parameters measured in an objective way. In this manner we could suggest that in vivo the best spermatozoa would comprise one "elite" in the journey through the fertilization site.

Keywords: Spermatozoa, Morphology, Strict criteria, Sperm motility, Sperm Concentration, Density gradients. 
$\mathrm{L}$ a infertilidad es un problema que afecta alrededor de 1 de cada 6 parejas, de las cuales aproximadamente un $50 \%$ puede deberse al factor masculino ${ }^{1}$. Varias etiologías han sido identificadas como posibles causa de la infertilidad masculina, entre éstas se encuentran: las mutaciones, las aneuploidias, las enfermedades infecciosas, la oclusión de ductos eyaculatorios, la presencia de varicocele, la exposición a irradiación, la quimioterapia y la disfunción eréctil. Sin embargo un gran porcentaje (40-50\%) de pacientes resultan infértiles idiopáticos ${ }^{2-8}$.

Uno de los problemas principales en el intento de identificar la causa de la infertilidad masculina es la dificultad para realizar el diagnóstico. La herramienta principal para tal fin es el análisis seminal o espermograma. Sin embargo existen evidencias que muestran que al evaluar los parámetros seminales en una población de individuos con fertilidad probada, al menos el $55 \%$ presentaban un parámetro alterado respecto a los valores de referencia establecidos por la Organización Mundial de la Salud (OMS) $)^{9,10}$. Adicionalmente, algunas parejas conciben rápidamente a pesar de que sus resultados en el espermograma son anormales según OMS y por el contrario, hombres con un análisis de semen normal son incapaces de embarazar a sus parejas. Debido a esto se ha postulado la necesidad de realizar estudios funcionales y bioensayos que brinden mayor información de la capacidad fecundante del espermatozoide tales como: la evaluación de la reacción acrosomal inducida, la evaluación de la hiperactivación, la prueba de unión a hemizona, prueba de penetración de la zona y ensayos de penetración de oocitos de hamster libres de zona pelúcida ${ }^{11}$. Sin embargo generalmente estas pruebas son sólo utilizadas en laboratorios de investigación ya que son engorrosas, costosas y difíciles de estandarizar entre laboratorios clínicos.

Debido a la necesidad de buscar un marcador que permita identificar en forma simple y confiable que individuos tienen mejores posibilidades de fecundación, surge el criterio de la morfología estricta como uno de los más importantes marcadores de la capacidad fecundante, al menos in vitro $^{9,12}$. La evaluación de la morfología espermática ha sido correlacionada con la capacidad de los espermatozoides para unirse a la zona pelúci- da ${ }^{13-16}$, y sobrellevar la reacción acrosomal ${ }^{17,18}$. Los espermatozoides anormales producirian especies reactivas de oxígeno las cuales dañarian el ADN celular por stress oxidativo ${ }^{19,20}$.

La cantidad de formas normales esta relacionada con el éxito en la fecundación in vitro ya que los pacientes con morfología $>14 \%$ tienen mejores posibilidades de fecundación de in vitro, mientras que aquellos por debajo del $4 \%$ son aconsejados para realizar inyección intracitoplasmática de espermatozoides (ICSI) ${ }^{12,21}$.

Con el advenimiento de los sistemas computarizados de análisis de movimiento espermático (CASA), se ha podido identificar y evaluar no solo el número de células móviles sino también analizar su trayectoria. Estos estudios permiten un análisis seminal más objetivo de la cinética espermática, cuyos parámetros han sido relacionados también con las posibilidades de éxito en fecundación in vitro ${ }^{22}$.

Debido a la necesidad de entender si los espermatozoides morfológicamente mejores son más veloces, el objetivo del presente trabajo consistió en evaluar si existe asociación entre el \% de formas normales y las variables de movimiento analizadas en forma objetiva.

\section{MATERIALES Y MÉTODOS}

Obtención de las muestras y análisis basal del semen

Se incluyeron las muestras de semen de pacientes que recurrieron para evaluación al Laboratorio Reprolab-Biología de la Reproducción del Sanatorio Británico de Rosario, Argentina. Las mismas se obtuvieron por masturbación en contenedores plásticos estériles y se conservaron durante $30-60$ min a $37^{\circ} \mathrm{C}$ para su licuefacción total. Sólo aquellas muestras de semen que presentaron $>20 \times 10^{6} \mathrm{esp} / \mathrm{ml}$ y $>50 \%$ de motilidad progresiva, de acuerdo a los parámetros de la OMS $^{9}$, fueron incluidas en este estudio $(n=203)$. Las muestras de 168 pacientes fueron divididas según el \% de formas normales en tres grupos: a) $<4 \%(\mathrm{n}=22), \mathrm{b})$ entre 4 y $13 \%(\mathrm{n}=89)$ y c) $\geq 14 \%$ $(\mathrm{n}=57)$.

\section{Gradiente de densidad}

Con el objetivo de concentrar los espermatozoides móviles y ver si esto se asociaba a una 
mejor morfología, se procesaron 35 muestras usando un gradiente de selección. La fracción inferior del gradiente (90\%) se preparó mezclando $9 \mathrm{ml}$ de solución isotónica de PureSperm, al 100\% con $1 \mathrm{ml}$ de medio Fluido Tubal Humano con HEPES, HTFm (GIBCO, BRL, Life Technologies Inc., Grand Island, NY, USA). Las fracciones superiores $(70 \%$ y $50 \%)$ se obtuvieron por dilución con HTFm de la solución al 90\%. A cada muestra se la separó en dos partes y se las colocó en gradientes paralelos que consistían en capas de 1,5 ml de cada solución y ambos tubos se centrifugaron a $400 \mathrm{~g}$ durante $20 \mathrm{~min}$. Posteriormente se descartó el plasma seminal y las fracciones de 50 y $70 \%$. La fracción de $90 \%$ se resuspendió en un tubo y se lavó con $2 \mathrm{ml}$ de HTFm a $400 \mathrm{~g}$ por $10 \mathrm{~min}$. Tanto la evaluación cinética como la determinación del \% de formas normales se realizaron antes y después del procedimiento de selección.

Análisis computarizado del movimiento (CASA)

Se tomó una gota $(5 \mathrm{ml})$ del semen basal o post recuperado, y se la colocó en una cámara de Makler (Sefi- Medical Instruments, Haifa, Israel). Las muestras se analizaron con un sistema computarizado de análisis de semen (MEII, RoyCo SA, Madrid, España) y se determinaron los siguientes parámetros: concentración de espermatozoides, $\%$ de espermatozoides móviles, \% de espermatozoides rápidos $>25 \mathrm{~mm} / \mathrm{s}$ (RAP,\%), velocidad media promedio (VAP, $\mathrm{mm} / \mathrm{s}$ ), linealidad media (LIN; VSL/VCL x 100\%), y desplazamiento lateral de la cabeza ( $\mathrm{LDH}, \mathrm{mm})$.

\section{Evaluación de la morfología}

Los extendidos tanto del semen basal como post separado se prepararon deslizando una gota de la muestra de manera de obtener una única película de grosor uniforme. Posteriormente se dejaron secar al aire, se fijaron con metanol (Anedra, Buenos Aires, Argentina) y se tiñeron con una solución de Hematoxilina "activada" (Solución según Gill № 2, Biopur S.R.L., Rosario, Argentina) $30 \% \mathrm{v} / \mathrm{v}$ en agua destilada. La morfología espermática se evaluó siguiendo el criterio estricto de Tygerberg ${ }^{12}$, se contaron al menos 100 espermatozoides utilizando objetivo de inmersión 100x, bajo observación micrométrica.

\section{Análisis estadístico}

El análisis estadístico se realizó utilizando el programa GraphPad InStat (GraphPad Software, San Diego, CA, USA). Los datos se expresaron como la media \pm el error estándar de la media (SEM) y se analizaron las diferencias entre los tres grupos usando las pruebas de ANOVA y Tukey-Kramer. Para comparar los datos antes y después de la selección se utilizó el test $t$ de Student para datos apareado. Una $\mathrm{p}<0,05$ se consideró estadísticamente significativa.

\section{RESULTADOS}

Es importante destacar que todos los pacientes presentaban una concentración y movilidad normal como criterio de inclusión por lo cual la única variable para la división en grupos, fue el \% de formas normales. El valor medio entre los tres grupos fue de: a) $2 \pm 0,2 \%(n=22)$, b) $8 \pm 0,3 \%(n=89)$ y c) $17 \pm 0,5 \%(n=57)$, respectivamente $(\mathrm{p}<0,0001)$.

Nuestros resultados muestran que mientras la concentración media en el grupo con $<4 \%$ resultó significativamente menor que el grupo con mejor morfología $\geq 14 \%\left(61 \pm 8,3 \times 10^{6} \mathrm{esp} / \mathrm{ml}\right.$ vs. $91 \pm 5,5$ x $10^{6} \mathrm{esp} / \mathrm{ml}$, respectivamente, $\left.\mathrm{p}<0,05\right)$, el $\%$ de formas móviles fue aumentando en forma significativa $(\mathrm{p}<0.05)$ a medida que aumenta el \% de espermatozoides normales entre los tres grupos (Fig. 1).

El análisis cinético reveló que tanto el \% de espermatozoides rápidos ( $>25 \mu \mathrm{m} / \mathrm{s})$ así como la velocidad media (VAP) y el desplazamiento de la cabeza (LHD) también se vieron incrementados por la cantidad de espermatozoides normales presentando los valores más bajos en el grupo < $4 \%$ respecto al grupo $\geq 14 \%(\mathrm{p}<0,05)$. La LIN se mantuvo constante entre los grupos (Fig. 2).

Cuando los espermatozoides móviles fueron seleccionados mediante un gradiente de densidad se obtuvieron los resultados que se muestran en la Tabla 1 donde vemos que luego del procedimiento todas las variables se vieron incrementadas respecto al valor basal, excepto la LIN. Adicionalmente, la tasa de recuperación de espermatozoides progresivos después del método de selección fue del $54 \pm 5.8 \%$.

Los datos obtenidos al dividir las muestras según el \% de formas normales en el semen muestran que en ambos grupos luego del gradiente, se incrementa significativamente el \% de formas normales (Tabla 2). 


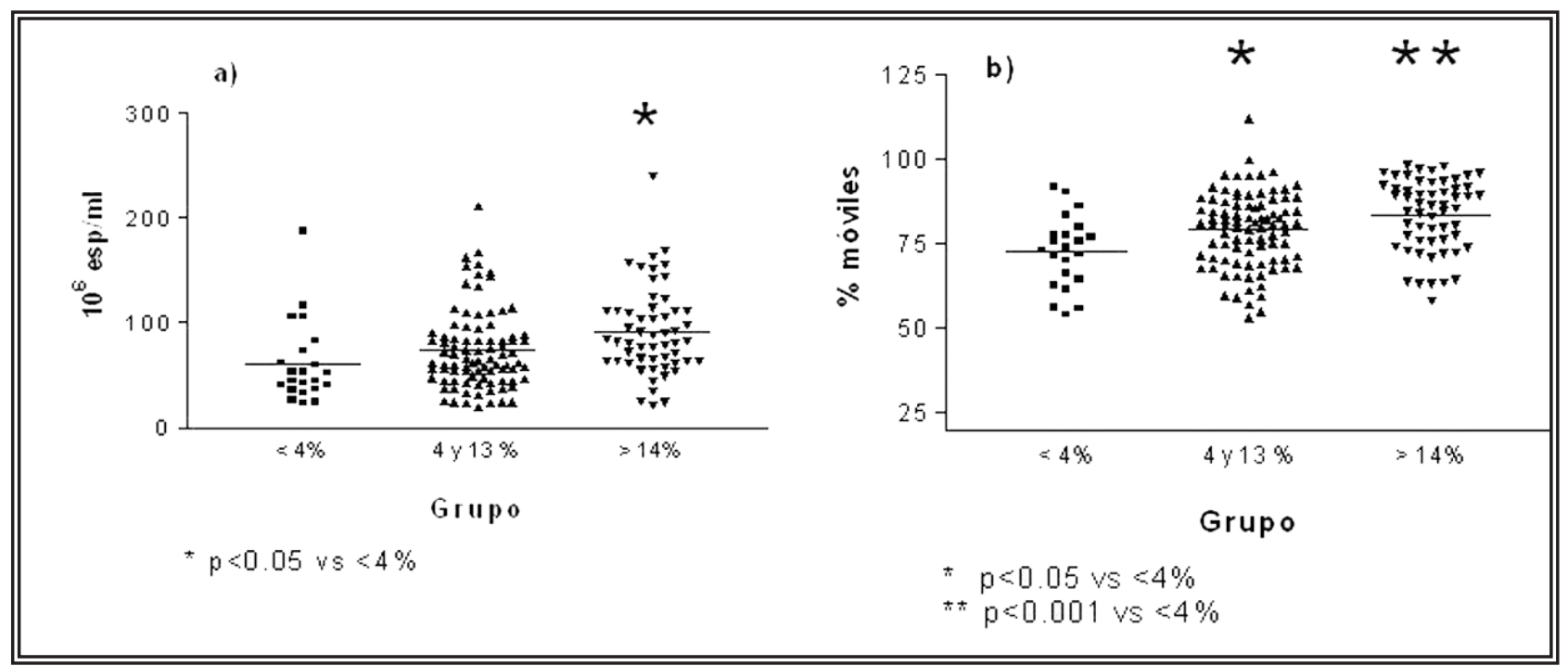

FIGURA 1. Relación entre el \% de formas normales, la concentración y la movilidad

Distribución de los datos al dividir los grupos según el \% de formas normales, < $4 \%$ ( $n=22$ ), entre 4 y $13 \%$ ( $n=89$ ) y $\geq a$ $14 \%(n=57)$ y compararlos tanto con a) la concentración como con b) la movilidad.

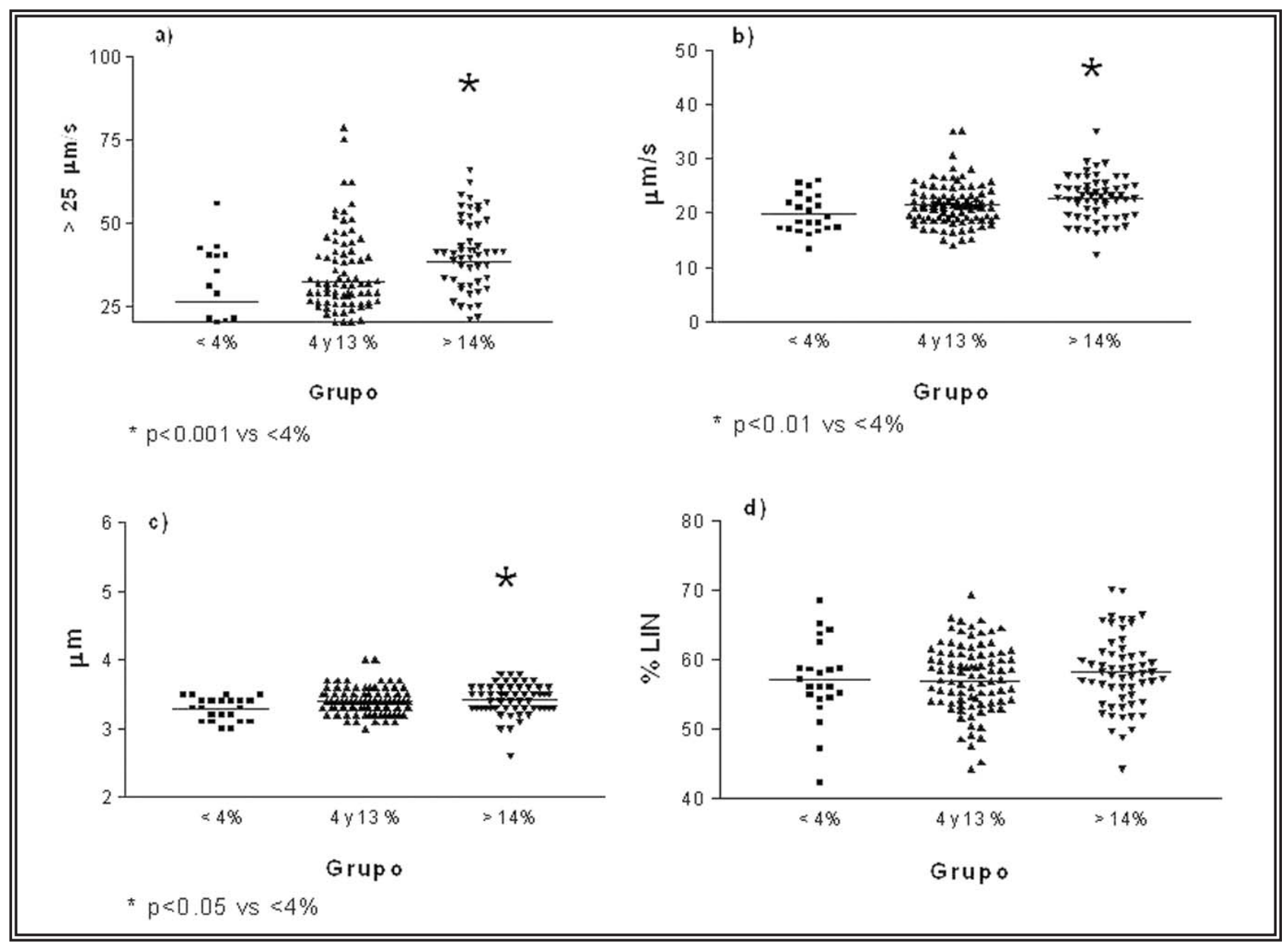

FIGURA 2. Relación entre el \% de formas normales y las características de desplazamiento

Distribución de los datos al dividir los grupos según el \% de formas normales, < 4\% ( $n=22)$, entre 4 y $13 \%$ ( $n=89$ ) y $\geq a$ $14 \%$ ( $n=57$ ) y compararlos tanto con a) \% RAP, \% de rápidos ( $25 \mu \mathrm{m} / \mathrm{s}) ;$ b) VAP ( $\mathrm{Hm} / \mathrm{s})$, velocidad media promedio; c) LHD $(\mu \mathrm{m})$, desplazamiento lateral de la cabeza como con d) LIN, linealidad media. 
Tabla 1

Parámetros seminales básales y post selección $(n=35)$

\begin{tabular}{lccc}
\hline Variable & $\begin{array}{c}\text { Basal } \\
\text { media } \pm \text { ds }\end{array}$ & $\begin{array}{c}\text { Post separación } \\
\text { media } \pm \text { ds }\end{array}$ & Valor de p \\
\hline Concentración (x 106 esp/ml) & $69,7 \pm 7,1$ & $154,0 \pm 16,5$ & $<0,001$ \\
Movilidad Progresiva $(25 \mu \mathrm{m} / \mathrm{s}, \%)$ & $59,7 \pm 3,0$ & $86,5 \pm 2,8$ & $<0,0001$ \\
Células Rápidas (\%) & $27,4 \pm 2,6$ & $51,6 \pm 19,8$ & 0,0007 \\
VAP (mm/s) & $20,5 \pm 0,8$ & $28,7 \pm 1,0$ & $<0,0001$ \\
LIN (\%) & $59,5 \pm 1,3$ & $58,5 \pm 1,8$ & $0,09 \mathrm{NS}$ \\
LHD (mm) & $3,4 \pm 0,05$ & $3,8 \pm 0,06$ & $<0,0001$ \\
Espermatozoides normales (\%) & $19,0 \pm 1,5$ & $41,9 \pm 2,6$ & $<0,0001$ \\
\hline
\end{tabular}

VAP, velocidad media promedio; LIN, linealidad media; LHD, desplazamiento lateral de la cabeza. NS: no significativo.

Tabla 2

Formas normales en semen basal vs. post selección

\begin{tabular}{cccc}
\hline Clasificación & $\begin{array}{c}\text { Basal } \\
\text { media } \pm \text { ds }\end{array}$ & $\begin{array}{c}\text { \% formas normales } \\
\text { Post separación } \\
\text { media } \pm \text { ds }\end{array}$ & valor de p \\
\hline$\geq 14$ & $23,0 \pm 1,0$ & $45,7 \pm 2,9$ & $<0,0005$ \\
$<14$ & $10,3 \pm 0,7$ & $33,5 \pm 4,1$ & $<0,0002$ \\
\hline
\end{tabular}

\section{DISCUSIÓN}

Si bien los avances vertiginosos de la última década con el advenimiento del ICSI, parecerían dejar de lado la necesidad de comprender profundamente el mecanismo exacto que lleva a la interacción entre ambas gametas, no resulta ser un dato menor al la hora de intentar la concepción natural o al utilizar tratamientos menos complejos tales como la Inseminación Intrauterina (IIU) o la FIV $^{23}$. Por lo tanto, el objetivo del presente estudio ha sido evaluar si existe asociación entre la morfología normal del espermatozoide y su cinética de desplazamiento.

Es importante destacar que en este trabajo todos los pacientes incluidos tenían número y \% de móviles de acuerdo a los valores de normalidad de $\mathrm{OMS}^{9}$, siendo el \% de formas normales la única variable entre grupos. Se observa una estrecha asociación entre el grupo de peor pronóstico (morfología $<4 \%$ ) y las valores más bajos en las variables cinéticas. En un estudio previo ${ }^{24}$ se mostró que tanto la morfología como las variables cinéticas evaluadas por el CASA predecían el resultado de la IIU, sin embargo en ese trabajo todos los pacientes presentaban análisis seminales alterados. Por lo tanto es bastante importante recalcar que aunque todas las muestras de nuestro estudio presentaban concentración nor- mal, al dividir los grupos según la morfología y hacer el análisis se observa que a mejor morfología mejor es la concentración espermática $(\mathrm{p}<0,05)$.

Nuestros datos muestran que un incremento en el \% de espermatozoides rápidos $(>25$ $\mathrm{mm} / \mathrm{s})$ y \% de móviles a medida que aumentaba la cantidad de formas normales. La LIN se mantuvo constante entre los grupos hecho que podría explicarse considerando que aunque existe un aumento en la velocidad espermática (VCL), también se incrementa la velocidad en línea recta de la trayectoria (VSL), lo que implica que la LIN que resulta del cociente de ambos parámetros (LIN; VSL/VCL x 100\%), permanezca homogénea entre grupos.

El avance de las técnicas de reproducción asistida tales como la IIU o la FIV, ha llevado a desarrollar métodos de selección de espermatozoides con el fin de aumentar la proporción de espermatozoides móviles con morfología normal, libres de plasma seminal, desechos celulares, leucocitos y bacterias $^{25}$. Esto ha sido claramente demostrado en nuestros resultados ya que la población de espermatozoides seleccionados presentó mejor velocidad y concentración así como cantidad de formas normales. Nuestro trabajo muestra que mediante el uso de gradientes el sistema puede rescatar prácticamente $50 \%$ de espermatozoides progresivos, dato que resulta comparable con el $46 \%$ reportado por Claassens et al. en un grupo similar de pacientes ${ }^{26}$. Luego la selección aumenta tanto la VAP y el LHD sin que se manifieste diferencias en la LIN. Esto debido quizás a que los espermatozoides son recuperados del plasma seminal iniciándose el proceso de capacitación, que se asociaría con un movimiento vigoroso de gran desplazamiento lateral de la cabeza y baja linealidad llamado "hiperactivación" 27,28 . El hecho de que después de pasar por el gradiente los espermatozoides presenten 
mayor velocidad y LHD, podría relacionarse con etapas tempranas de la capacitación asociadas a la hiperactivación la cual alcanza valores máximos dentro de la hora haber removido el plasma seminal $^{29}$.

Se ha visto que los espermatozoides seleccionados presentan mayor proporción de acrosomas intactos ${ }^{30}$, mejores tasas de penetración de oocito de hámster ${ }^{31}$, aumento en el \% de FIV ${ }^{32}$ además de una reducción considerable en los niveles de especies reactivas del oxígeno ${ }^{33}$. Nuestros datos estarían de acuerdo con lo presentado por Hirano Y. et al. ${ }^{22}$ ya que en ambos se muestra que la selección por gradiente produce un incremento en la cantidad de espermatozoides y en la calidad de desplazamiento de los mismos, así como en el número de células con morfología normal, resultados similares son obtenidos usando swim-up.

Un trabajo previo de Hauser et al. ${ }^{34}$ mostró que la tasa de IIU es mayor en el grupo con mejor $\%$ de formas normales. Sin embargo, la metodología usada por el grupo para preparar la muestra para IIU fue una doble centrifugación, mediante la cual se pasa de un \% de formas normales de $9.1 \%$ en la muestra inicial a solamente $10.4 \%$ en la muestra preparada. En nuestro trabajo, los valores obtenidos post selección son estadísticamente diferentes a los del semen inicial aún en el grupo de $<14 \%$ de formas normales $(\mathrm{p}<0,0005)$. Por lo tanto nuestros datos apoyarian la utilización de gradientes de selección con el fin de aumentar el \% de espermatozoides normales (y las posibilidades de fecundar) aún en pacientes teratozoospérmicos moderados $(<14 \%)^{12}$. Es de destacar que como es sabido pacientes en teratozoospérmicos severos $(<4 \%)$, sus posibilidades aún de éxito en fecundación in vitro son escasas por lo que consideramos indicado el sugerirles ICSI. Esto indicaría que si bien la técnica de selección es un gradiente artificial podríamos suponer que actuaría "simulando" el paso por el cérvix y por lo tanto seleccionando los mejores espermatozoides lo cual aumenta las posibilidades de fecundar ${ }^{35}$.

Fisiológicamente, millones de espermatozoides son depositados mediante el eyaculado en la vagina, cientos de estos entran al oviducto y solo unos pocos interactúan con el oocito para que finalmente sólo uno pueda fecundarlo. Durante todo este paso de los espermatozoides por el tracto reproductivo femenino se requiere que los espermatozoides sufran una serie de cambios conocidos como capacitación que lo preparan para fecundar ${ }^{27}$. Existen distintas etapas de selección espermática que hacen que solo una "élite" de espermatozoides logre llegar al sitio de fecundación ${ }^{35}$, gracias a su capacidad de movimiento ${ }^{36}$ y a sus mejores características morfológicas $^{35}$.

Con base en lo anterior, podemos pensar que los espermatozoide que tienen mejor morfología, serían los que aerodinámicamente tendrían el área adecuada y por lo tanto menor fricción con medio (ya sea plasma seminal, moco cervical o los distintos microambientes del tracto femenino) haciéndolos menos resistentes. Debido a esto tendrían una mejor calidad de desplazamiento, contrario a lo que se supone sucedería con los espermatozoides morfológicamente anormales ${ }^{37}$.

Si bien nuestros datos apoyan la idea de que evaluar la movilidad de un modo objetivo, puede pronosticarnos la presencia de una subpoblación de espermatozoides con mejores características morfológicas, no consideramos apropiado pensar que la evaluación de una característica pudiese reemplazar a la otra. Por el contrario defendemos la necesidad de realizar la evaluación morfológica en forma exhaustiva y micrométrica, poniendo límites claros para detección de los espermatozoides "normales".

\section{REFERENCIAS}

1. Irvine DS. Epidemiology and aetiology of male infertility. Hum Reprod. 1998;13(13):33-44.

2. Wang A, Fanning L, Anderson DJ, Loughlin KR. Generation of reactive oxygen species by leukocytes and sperm following exposure to urogenital tract infection. Arch Androl 1997;39(1):11-17.

3. Padron OF, Brackett NL, Sharma RK, Lynne CM, Thomas AJ Jr., Agarwal A. Seminal reactive oxygen species and sperm motility and morphology in men with spinal cord injury. Fertil Steril. 1997;67(6):1115-1120.

4. Padron OF, Sharma RK, Thomas AJ Jr., Agarwal A. Effects of cancer on spermatozoa quality after cryopreservation: a 12-year experience. Fertil Steril. 1997;67(2):326-331.

5. Carbone DJ Jr., Shah A, Thomas AJ Jr., Agarwal A. Partial obstruction, not antisperm antibodies, causing infertility after vasovasostomy. J Urol. 1998; 159:827-830.

6. Hendin BN, Kolettis PN, Sharma RK, Thomas AJ Jr., Agarwal A. Varicocele is associated with elevated spermatozoal reactive oxygen species production and diminished seminal plasma antioxidant capacity. J Urol. 1999;161:1831-1834. 
7. Mak V, Zielenski J, Tsui LC, Durie P, Zini A, Martin S, et al. Proportion of cystic fibrosis gene mutations not detected by routine testing in men with obstructive azoospermia. Jama. 1999;281(23):2217-2224.

8. Ollero M, Gil-Guzman E, Lopez MC, Sharma RK, Agarwal A, Larson K, et al. Characterization of subsets of human spermatozoa at different stages of maturation: implications in the diagnosis and treatment of male infertility. Hum Reprod. 2001;16(9):1912-1921.

9. WHO. WHO Laboratory Manual for the Examination of Human Semen and Sperm-Cervical Mucus Interaction. Cambridge: Cambridge University Press. 1999.

10. De los Ríos J, Cardona WD, Berdugo JA, Correa C, Arenas A, Olivera-Angel M, et al. Los valores espermáticos de 113 individuos con fertilidad reciente no mostraron correlación con los parámetros establecidos por la OMS. Arch Esp Urol. 2004;57(2):147-152.

11. Oehninger S. An update on the laboratory assessment of male fertility. Hum Reprod. 1995;10(1):38-45.

12. Kruger TF, Acosta AA, Simmons KF, Swanson RJ, Matta JF, Oehninger S. Predictive value of abnormal sperm morphology in in vitro fertilization. Fertil Steril. 1988;49 (1): 112-117.

13. Menkveld R, Franken DR, Kruger TF, Oehninger S, Hodgen GD. Sperm selection capacity of the human zona pellucida. Mol Reprod Dev. 1991;30(4):346-352.

14. Liu DY, Baker HW. The proportion of human sperm with poor morphology but normal intact acrosomes detected with Pisum sativum agglutinin correlates with fertilization in vitro. Fertil Steril. 1988;50(2):288-293.

15. Liu DY, Baker HW. Morphology of spermatozoa bound to the zona pellucida of human oocytes that failed to fertilize in vitro. J Reprod Fertil. 1992;94:71-84.

16. Menkveld R, Rhemrev JP, Franken DR, Vermeiden JP, Kruger TF. Acrosomal morphology as a novel criterion for male fertility diagnosis: relation with acrosin activity, morphology (strict criteria), and fertilization in vitro. Fertil Steril. 1996;65(3):637-644.

17. Franken DR, Bastiaan HS, Kidson A, Wranz P, Habenicht UF. Zona pellucida mediated acrosome reaction and sperm morphology. Andrologia. 1997;29(6):311-317.

18. Menkveld R, El-Garem Y, Schill WB, Henkel R. Relationship between human sperm morphology and acrosomal function. J Assist Reprod Genet. 2003;20(10):432-438.

19. Aitken RJ, Gordon E, Harkiss D, Twigg JP, Milne P, Jennings $Z$, et al. Relative impact of oxidative stress on the functional competence and genomic integrity of human spermatozoa. Biol Reprod. 1998;59(5):1037-1046.

20. Ford WC. Regulation of sperm function by reactive oxygen species. Hum Reprod Update 2004;10(5):387-399.

21. ESHRE. Manual on Basic Semen Analysis; 2002. editors, Kvist U y Björndahl L. Oxford university Press.

22. Hirano Y, Shibahara H, Obara H, Suzuki T, Takamizawa S, Yamaguchi C, et al. Relationships between sperm motility characteristics assessed by the computer-aided sperm analysis (CASA) and fertilization rates in vitro. J Assist Reprod Genet. 2001;18(4):213-218.

23. Dickey RP, Pyrzak R, Lu PY, Taylor SN, Rye PH. Comparison of the sperm quality necessary for successful intrauterine insemination with World Health Organization threshold values for normal sperm. Fertil Steril 1999;71(4):684-689.
24. Shibahara H, Obara H, Ayustawati, Hirano Y, Suzuki T, Ohno A, et al. Prediction of pregnancy by intrauterine insemination using CASA estimates and strict criteria in patients with male factor infertility. Int $\mathrm{J}$ Androl. 2004; 27(2):63-68.

25. Levay PF, Fourie FR, Bezuidenhout C, Koch A. Effectiveness of various sperm processing methods in removing seminal plasma from insemination media. Hum Reprod. 1995;10(8):2056-2060.

26. Claassens OE, Menkveld R, Harrison KL. Evaluation of three substitutes for Percoll in sperm isolation by density gradient centrifugation. Hum Reprod. 1998;13:3139-3143.

27. Yanagimachi R. Mammalian Fertilization. In: Neill EKaJ, editor. The Physiology of Reproduction. New York: Raven Press; 1994;189-317.

28. Burkman LJ. Discrimination between nonhyperactivated and classical hyperactivated motility patterns in human spermatozoa using computerized analysis. Fertil Steril. 1991;55(2):363-371.

29. Aitken RJ. Cell biology of human spermatozoa. In: Grudzinskas JG y Yovich JL, editor. Gametes, The Spermatozoa. Cambridge University Press; 1995;206-237.

30. Brandeis VT, Manuel MT. Effects of four methods of sperm preparation on the motile concentration, morphology, and acrosome status of recovered sperm from normal semen samples. J Assist Reprod Genet. 1993;10(6):409-416.

31. Anphaichitr N, Millette CF, Agulnick A, Fitzgerald LM. Eggpenetration ability and structural properties of human sperm prepared by Percoll-gradient centrifugation. Gamete Res 1988;20(1):67-81.

32. Jaroudi KA, Carver-Ward JA, Hamilton CJ, Sieck UV, Sheth KV. Percoll semen preparation enhances human oocyte fertilization in male-factor infertility as shown by a randomized cross-over study. Hum Reprod. 1993;8(9): 1438-1442.

33. Aitken RJ, Clarkson JS. Significance of reactive oxygen species and antioxidants in defining the efficacy of sperm preparation techniques. J Androl. 1988;9(6):367-376.

34. Hauser R, Yogev L, Botchan A, Lessing JB, Paz G, Yavetz $H$. Intrauterine insemination in male factor subfertility: significance of sperm motility and morphology assessed by strict criteria. Andrologia 2001;33(1):13-17.

35. De Jonge C. Biological basis for human capacitation. Hum Reprod Update. 2005;11(3):205-214.

36. Olds-Clarke P. How does poor motility alter sperm fertilizing ability? J Androl. 1996;17(3):183-186.

37. Shi YD, Pan LF, Yang FK, Wang SQ. A preliminary study on the rheological properties of human ejaculate and changes during liquefaction. Asian J Androl. 2004;6(4): 299-304.

Dra. $\mathrm{M}^{\mathrm{a}} \mathrm{J}$. Munuce

Sanatorio Británico de Rosario y Servicio

de Reproducción Humana y Planificación Familiar

San Lorenzo 939-9A

2000 Rosario - Argentina

E-mail: reprolab@citynet.net.ar

(Trabajo recibido el 12 de diciembre 2005) 